\author{
Dirk Beckedorf
}

Franz Müller

\section{Einführung und Übersicht}

Zwischen 1950 und 1960 beschäftigte sich der französische HalsNasen-Ohrenarzt Alfred Tomatis intensiv damit, die Zusammenhänge zwischen Gehör, Stimme und Psyche zu erforschen. Dabei spielte die Analyse des Gesangs von Enrico Caruso, der grossen, goldenen Stimme des 20. Jahrhunderts, eine wichtige Rolle. Tomatis kam zu dem Schluss, dass ein bevorzugtes Hören mittlerer und hoher Töne die Stimme voller und heller erklingen lässt und darüber hinaus auch auf psychischer Ebene ein Ausdruck von einem «Hören-Wollen» und gelingender Kommunikation sein kann. Aktuelle neurobiologische Erkenntnisse, im Besonderen die Polyvagaltheorie des amerikanischen Forschers Stephen Porges, belegen diese Aussage und geben ihr eine erweiterte Bedeutung: Zuhören, sichere Bindung und ein ruhiger Zustand des unbewussten Nervensystems gehen miteinander einher. Eine zentrale Bedeutung hat dabei ein besonderer Anteil des Nervus vagus, des "grossen Ruhenervs». Seine Aktivierung fördert eine achtsame Haltung zu uns selbst und gegenüber anderen.

\section{Die Tomatis-Gesetze und die Stimme von Enrico Caruso}

Dr. Tomatis arbeitete in den 1950erJahren als klassischer Hals-NasenOhrenarzt in Paris. Sein Vater, ein Opernsänger, schickte Kollegen mit stimmlichen Problemen zu seinem Sohn. Tomatis stellte durch übliche Untersuchungen wie Kehlkopfspiege-

\title{
Enrico Caruso, Alfred Tomatis und die moderne Neurobiologie - prosoziales Hören, ruhiger Herzschlag und gelingende Kommunikation
}

lung fest, dass einige dieser Sänger keine fassbare körperliche Ursache ihrer Stimmstörungen hatten. Er kam auf einen damals ungewöhnlichen Gedanken: Er untersuchte das Frequenzspektrum der Stimme und verglich es anschliessend mit dem Hörvermögen. Es zeigte sich eine überraschende Übereinstimmung: Töne, die vermindert gehört wurden, fehlten auch in der Stimme [1]. Diese Erkenntnisse wurden in einem ersten Tomatis-Gesetz formuliert und später durch andere Untersucher bestätigt: Die Stimme enthält als Obertöne die Frequenzen nur in dem Umfang, wie sie das Ohr wahrnimmt. Als ein Beispiel kann die durchschnittliche Abnahme der Hörfähigkeit in hohen Frequenzen bei älteren Menschen angeführt werden. Bei den Betroffenen sind als Folge die Obertöne ihrer Stimme vermindert.

Tomatis konstruierte im Folgenden einen Apparat mit Verstärkern und Filtern. Er liess die Sänger mit Stimmstörungen in ein Mikrofon singen, verstärkte die schlecht gehörten Frequenzen und liess sie nun in Echtzeit über Kopfhörer ihre eigene, verbesserte Stimme hören. Das Ergebnis war, dass sofort und unmittelbar die vorher verminderten oder fehlenden Töne wieder in der Stimme auftauchten. Diese Experimente bewiesen den Regelkreis zwischen Gehör und Stimme und mündeten im zweiten Tomatis-Gesetz: Gibt man dem Gehör Gelegenheit, schlecht gehörte Frequenzen wieder normal zu hören, tauchen diese sofort und unbewusst wieder in der Stimme auf. Aufbauend auf diesen ersten Untersuchungen wurde nun ein Therapiegerät zur Konditionierung des Gehörs entwickelt, das «Elektronische Ohr».

Tomatis fragte sich nun weiter, wie denn ein für die Stimme und im erweiterten Sinn auch allgemein für Kommunikation «ideales» Gehör beschaffen sein sollte. Er analysierte die Stimme von Enrico Caruso, dessen Klang in besonderem Masse die Herzen der Menschen berühren konnte. Durch Reihenuntersuchungen hatte man zuvor bereits in den 1940er-Jahren herausgefunden, dass das menschliche Gehör besonders empfindlich im Tonbereich zwischen 1000 und $5000 \mathrm{~Hz}$ ist [2]. Sehr tiefe und auch sehr hohe Töne müssen erheblich lauter werden, bis wir sie überhaupt wahrnehmen können. Tomatis erkannte, dass Carusos Gehör in noch weit grösserem Ausmass diese mittleren und hohen Töne hervorhob. Aufgrund des oben beschriebenen Regelkreises von Gehör und Stimme bewirkte dieses besondere Gehör von Caruso seinen aussergewöhnlichen Reichtum von Obertönen, die besondere Strahlkraft seiner Stimme.

Die Frage tauchte nun auf: Wie haben Caruso bzw. sein Körper und Gehirn diese besondere Betonung im Hören hoher Frequenzen vollbracht? Eine wichtige Rolle spielen in den Erklärungen von Tomatis zwei kleine Muskeln, die die Spannung des Trommelfells weitgehend unwillkürlich regulieren (Abb. 1). Die meisten Leser dieses Artikels werden bislang vielleicht nichts von diesen Muskeln gewusst haben und doch benutzen sie sie

\section{KARGER}

Fax +497614520714
(๑) 2014 S. Karger GmbH, Freiburg

Accessible online at: www.karger.com/szg
Dr. med. Dirk Beckedorf

Neukirchstrasse 12, 28215 Bremen, Deutschland

dirk@drbeckedorf.de 


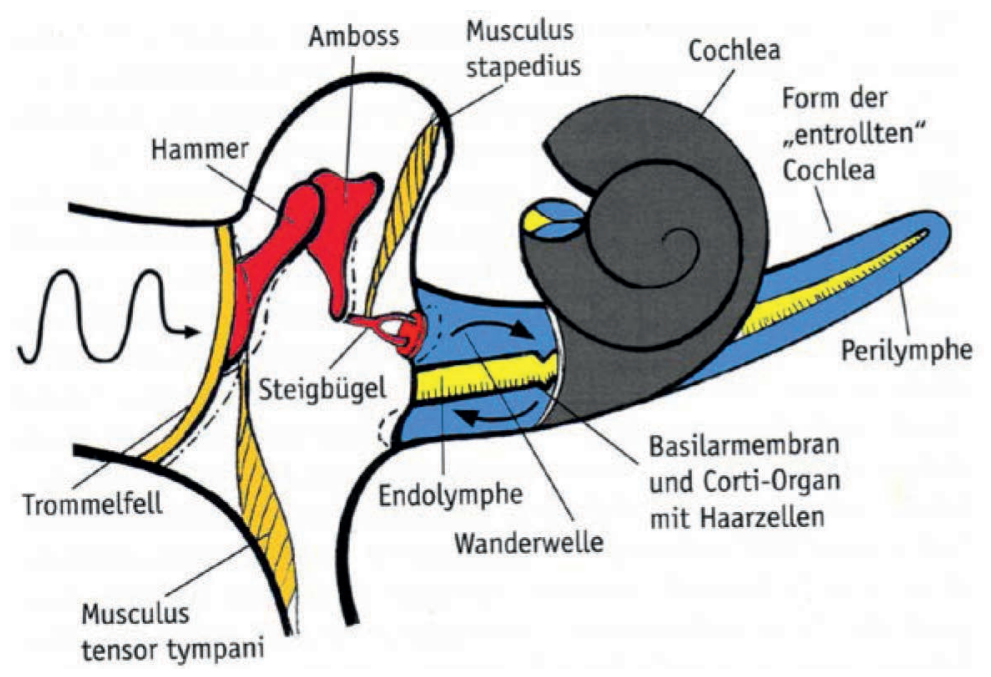

Abb. 1. Mittelohrmuskeln und Weiterleitung der Schallwellen zu Innenohr und Haarzellen.

immer dann, wenn etwas interessant, lusterweckend und neugierig machend klingt. Dann spannen diese Muskeln vom autonomen Nervensystem (ANS) gesteuert das Trommelfell, d.h., die Ohren werden «gespitzt». Vergleichbar mit einer gut gespannten Membran einer Trommel oder Pauke werden nun höhere Töne bevorzugt zum Innenohr und Gehirn geleitet. Wir werden aufmerksam, wach und richten uns auf. Zudem wird unser Herzschlag und Atemrhythmus in diesem Moment in ruhiger Weise aufeinander abgestimmt und das Gesicht bekommt eine lebendige Spannung.

Jahrzehnte nach Alfred Tomatis wurde diese weitgehend unbewusste Reaktion unserer Ohrmuskeln und unseres Nervensystems durch die Polyvagaltheorie des amerikanischen Neurophysiologen Stephen Porges bestätigt [3].

Über die Bedeutung der Polyvagaltheorie sagt der bekannte Traumatherapeut Bessel van der Kolk: «Es ist ein aussergewöhnliches Erlebnis, ein neues Musikstück oder eine neue wissenschaftliche Idee zu hören, etwas, dass ... in unserem Geist nicht nur ein Licht, sondern eine ganze Galerie von Lichtern aufleuchten lässt, die unser Verständnis von Sinn und Zweck des Lebens für immer verändert.» Hierzu zählt van der Kolk den Vortrag von Porges, in dem er 1999 die Polyvagaltheorie präsentierte [3, S. 11]. Seine
Forschungen revolutionierten die alten Auffassungen von den Funktionen des ANS und unterstützten die Ansichten vieler anderer Neurobiologen: Unser menschliches Nervensystem ist auf Kommunikation und Kontakt ausgerichtet. Wir sind soziale Wesen.

\section{Das autonome Nervensystem und die klassische Sicht einer Dualität von Parasympathikus und Sympathikus}

Das ANS ist ein entwicklungsgeschichtlich älterer Teil unseres Nervensystems. Es liegt weit unten im Gehirn und Rückenmark und seine feinen Nervenäste erreichen alle inneren Organe wie auch die Haut. Durch die Tätigkeit des ANS brauchen wir morgens beim Aufstehen nicht willentlich die Körpertemperatur der Umgebungsluft anpassen und wir müssen auch nicht die Menge der Magensäure für unser Frühstück bemessen. Auch die Intensität und Ausrichtung der Aufmerksamkeit sowie der Haut- und Muskeldurchblutung werden durch das ANS gesteuert. Als Beispiel soll folgende Szene dienen: Wir liegen an einem Sonntagnachmittag auf dem Sofa und dösen vor uns hin. Unsere Aufmerksamkeit ist niedrig und ungerichtet, mehr bei uns selbst und unserem Körper. Wir nehmen die Wärme der gut durch- bluteten Haut wohlig wahr. Plötzlich gibt es einen Krach, Scherben klirren und dann schreit unser Kind laut und schrill. Sofort sind wir hellwach, unsere Aufmerksamkeit ist aussen und auf die Ursache des Schreiens gerichtet. Der Herzschlag ist beschleunigt und wir springen auf, um die Ursache der Ereignisse zu klären.

In der klassischen Theorie besteht das ANS aus zwei grossen Ästen, die polar entgegengesetzt wirken und in der gerade beschriebenen Situation nacheinander tätig werden: der beruhigende Parasympathikus und der aktivierende Sympathikus. Der Sympathikus ist der Stressast, der den Körper und den Geist immer dann in Erregung versetzt, wenn das Gehirn eine Bedrohung, eine Unsicherheit oder ein ungelöstes Problem erkennt - wie im obigen Beispiel das laute klirrende Geräusch und das Kindergeschrei. Er hat zwei basale Möglichkeiten: Kämpfen oder Flüchten. Sein Name bedeutet «Mitleiden, Mitempfinden» [4]. Gefühle/Emotionen wurden ursprünglich vor allem dem Sympathikus zugeordnet [5]. Sein Gegenspieler, der beruhigende Parasympathikus, wird immer aktiv, wenn das Gehirn erkennt, dass innerlich und äusserlich alles überwiegend gut und sicher ist. Schon Darwin hat über die Funktion des Parasympathikus berichtet [6]. In der klassischen Sicht reguliert der Parasympathikus vor allem die Verdauung, beruhigt dann auch den Herzschlag und den Atem und ist im Schlaf aktiv. Etwas zugespitzt sah diese Auffassung des ANS in der biologischen Natur des Menschen im Wesentlichen die beiden Möglichkeiten: Verdauen und Schlafen oder Kämpfen und Flüchten.

In den vergangenen Jahrzehnten wurde immer mehr hervorgehoben, wie bedeutsam Anteile des Parasympathikus für die achtsame Wahrnehmung des eigenen Körpers und auch für die Kommunikation mit anderen Menschen ist. Eine besondere Bedeutung hat dabei der wichtigste Nerv 
des Parasympathikus, der Nervus vagus. Seine Name bedeutet der «Weitschweifende», und das ist bei diesem Nerv auch der Fall: Er verlässt das Gehirn in etwa auf Höhe der Ohren, gibt Äste zum Trommelfell ab, wandert auf beiden Seiten entlang der Speiseröhre nach unten und schickt einen Zweig zum Kehlkopf, um die Stimmlippen zu versorgen. Wir sprechen und singen also mithilfe des Nervus vagus. Dann schickt er Äste zum Herzen sowie zur Lunge und schiebt sich weiter auf beiden Seiten des Magens in die Bauchhöhle, um grosse Teile des Darms zu steuern. Er wird auch als "grosser Ruhenerv» [7] bezeichnet, da er Herzschlag und Atemrhythmus beruhigt und auch für die Abstimmung von Herzschlag und Atem aufeinander verantwortlich ist. Der Herzschlag hat in Abhängigkeit vom Atem eine natürliche Pulsation: Bei der Einatmung beschleunigt er sich etwas, bei der Ausatmung wird er etwas langsamer. Man kann dieses Phänomen mit zwei Zügeln vergleichen, die unser Herz in einem auf- und abschwellenden Rhythmus lenken. Diese pulsatorische Beziehung von Herzschlag und Atemfrequenz zählt zur Herzratenvariabilität. Sie ist ein Zeichen von Gesundheit: Bei Depressionen engt sich die Herzratenvariabilität ein, die Zügel werden bildlich gesprochen unter Dauerstress immer enger angezogen [8]. Dies könnte auch erklären, warum bei Depressionen das Risiko, einen Herzinfarkt zu bekommen, erhöht ist [9].

Eine hohe Aktivität des Nervus vagus ist also ein Zeichen von Gesundheit. Der Körper ist in einem Grundzustand von Sicherheit und Ruhe. Andererseits kann es sehr selten auch zu einem plötzlichen, abrupten und sehr starken Absinken des Herzschlags kommen, einer lebensbedrohlichen Bradykardie. Diese neurogene (nervlich vermittelte) Bradykardie wird auch über den Nervus vagus bewirkt. Wieso ist im einen Fall eine hohe Aktivität des Nervus vagus mit seiner pulsatorischen Herzratenvaria- bilität ein Zeichen von Gesundheit, während in anderen Fällen eine hohe Aktivität des Nervus vagus - wie bei der neurogenen Bradykardie - lebensbedrohlich werden kann? Dies wird auch als Vagusparadox [3, S. 23-25] bezeichnet und konnte bis vor einigen Jahren nicht richtig erklärt werden.

Ein weiteres körperliches Reaktionsschema gab bis vor einigen Jahren Rätsel auf: Beschrieben wurde oben, wie der Parasympathikus den Körper in einen Zustand von Entspannung und ruhiger Wachsamkeit versetzt. Gibt es Probleme, schaltet sich der Sympathikus mit seinen basalen Reaktionsmustern des Kämpfens oder Flüchtens ein. Immer wieder kommen Menschen leider auch in Situationen, die in Intensität und Dauer ausweglos erscheinen. Hierzu zählen Gewalterfahrungen im Krieg, sexualisierte Gewalt oder Unfälle. Körperlich kommt es häufig zu einer Erstarrungsreaktion bei gleichzeitig maximaler innerer Erregung. Es ist die letzte Möglichkeit, die die Natur Tier und Mensch lässt. Eine Antilope auf der Flucht vor dem Tiger geht in dem Moment, wenn sie keinen Ausweg mehr sieht, in den Totstellreflex [10]. Dies erfolgt auch bei Menschen im Erleiden eines seelischen Traumas. Es kommt dabei typischerweise zur Dissoziation, d.h., ein Teil der Seele begibt sich innerlich an einen Ort, wo er nicht mehr vom Geschehen berührt wird.

Wie aber wird diese Traumareaktion biologisch bewirkt? Es ist offenkundig etwas ganz anderes als die sympathikotone Aktivierung, sieht äusserlich scheinbar der parasympathischen Ruhe ähnlich und ist doch verbunden mit höchster Anspannung und Erregung.

\section{Die Polyvagaltheorie}

Eine fundamentale Erklärung für diese Phänomene gibt die Polyvagaltheorie von Stephen Porges, der ab Ende der 1990er-Jahre mit ihr an die Öffentlichkeit trat.
Hiernach gibt es in jedem Vagusnerv zwei Anteile: einen entwicklungsgeschichtlich älteren und einen entwicklungsgeschichtlich jüngeren Teil mit jeweils eigenen Nervenkerngebieten im Gehirn. Der ältere Teil heisst dorsaler Vagus (dorsal = zum Rücken, nach hinten liegend), der jüngere Teil wird als ventraler Vagus bezeichnet (ventral = zum Bauch, nach vorne liegend).

Der ältere, dorsale Vagus entstand in der Evolution mit den Reptilien. Reptilien haben eine dicke Haut. Bei neuen Reizen reagieren sie mit einem Moment des Verharrens, der Erstarrung, bevor sie sich orientiert haben und reagieren. In diesem Moment der orientierenden Erstarrung verlangsamt ihr Nervensystem über den dorsalen Vagus den Herzschlag, um Sauerstoff zu sparen. Diese Reaktion ist also für Reptilien adaptiv günstig.

Mit den Säugetieren entsteht eine völlig veränderte Lage: Säugetiere sind flink; sie waren kräftemässig den Reptilien meist unterlegen. Sie haben durch rasche Reaktion überlebt und mussten lernen, sich schnell $\mathrm{zu}$ orientieren und zu reagieren. Zudem haben sie in Relation zur Körpermasse einen fünfmal höheren Sauerstoffverbrauch als die Reptilien. Mit den Säugetieren entwickelte sich nun das jüngere, ventrale Vagussystem. Der ventrale Säugetiervagus beruhigt auch den Herzschlag; im Moment der Orientierung oder Gefahr wird aber diese «Vagusbremse» auf das Herz gelockert (genau andersherum als bei Reptilien).

Weiterhin wurde mit den Säugetieren die Haut weich und sensibel. Gefühle werden differenzierter und stimmliche Kommunikation wird wichtiger. Säugetiere wollen nicht nur wie die Reptilien in der Sonne sitzen und fressen, sondern wollen neben der Revierverteidigung und der Jagd auch kuscheln, sich reiben und spielen. Soziale Kommunikation wird immer bedeutsamer. Mit diesen Veränderungen entwickelt sich im Gehirn ein funktionaler Verbund von Nerven, 
der soziale Kommunikation ermöglicht. Porges nennt diesen Nervenverbund das «System Sozialen Engagements» (SSE) [3, S. 86]. Das SSE umfasst fünf der zwölf Hirnnerven unter anderem den Hirnnerv Nr. 5 (Nervus trigeminus), der die Gesichtshaut sensibel versorgt und auch die Kaumuskulatur sowie den Muskel steuert, der unwillkürlich das Trommelfell spannt. Mit dem Trigeminus fühlen wir also, wenn wir oder unser Partner über unser Gesicht streichen. Zum SSE gehört auch der Hirnnerv Nr. 7 (Nervus fascialis), mit dem wir unsere Gesichtsmuskeln bewegen und damit einen lebendigen Ausdruck auf unser Gesicht zaubern. Er steuert auch den zweiten Muskel hinter dem Trommelfell, den Stapediusmuskel. (Die Bedeutung dieser beiden Mittelohrmuskeln aus Sicht von Tomatis und Porges wird weiter unten näher ausgeführt.) Der dritte zum SSE zählende Nerv ist der Hirnnerv Nr. 9, der Glossopharyngeus, mit dem wir schlucken und die Muskeln der Mundhöhle bewegen. Er ist also für das Essen und Lautieren wichtig. Der vierte SSE-Nerv ist der Hirnnerv Nr. 10, Nervus vagus, über den bereits gesprochen wurde. Wichtig für das SSE ist sein ventraler Teil, der Säugetiervagus. Durch seine Aktivierung beruhigen sich der Herzschlag und die Atmung. Mittels seines Zweigs zu den Stimmlippen können wir unsere Stimme so modellieren, wie es unseren inneren Gefühlen entspricht. Und schliesslich zählt noch der Hirnnerv Nr. 11, der Nervus accessories, dazu, mit dem wir den Kopf wenden können und der uns damit eine rasche Reaktion auf auditive oder auch visuelle Reize ermöglicht.

Die fünf Nerven des SSE steuern somit alle für verbale und stimmliche Kommunikation wichtigen Muskeln.

Mit der Polyvagaltheorie wird aus der klassischen Dualität von Parasympathikus und Sympathikus ein Terzett, das die oben benannten Widersprüche (Vagusparadoxon und traumatische Erstarrungsreaktion) biologisch plausibel erklärt und zudem ein wesentlich differenzierteres, freundlicheres Bild von der menschlichen und auch tierischen Natur zeichnet.

Unser Gehirn unterscheidet drei Qualitäten der emotionalen Interaktion mit der Welt:

i) Sicherheit und Wohlbehagen;

ii) gravierendes Problem, Angst und Gefahr;

iii) Todesangst, Ohnmacht und Ausweglosigkeit.

In der ersten Qualität, dem Erleben von Sicherheit und Geborgenheit, werden der ventrale Vagus und das SSE aktiv. Der Körper ist in entspannter Wachsamkeit. Die Haut ist gut durchblutet. Die Gesichtsmimik ist in guter Grundspannung und bereit, lebendigen, freudigen Gefühlen Ausdruck zu verleihen. Der Herzschlag ist beruhigt und schwingt in Bezug zum Atem auf und ab. Die Stimmlippen sind bereit, in Spannung zu gehen, um zu tönen.

Dieses System des sozialen Engagements mit seinen fünf Nerven und der dazugehörenden Muskulatur ist mit der Geburt weitgehend funktionsfähig, die übrige Körpermuskulatur samt Nerven zum grossen Teil hingegen erst deutlich später. Ein neugeborenes Kind kann bei normaler Geburt nach wenigen Stunden den Kopf drehen. Es erkennt die Stimme seiner Mutter und kann selber mit seiner Stimme zeigen, ob es zufrieden ist oder schreiend auf Missstände aufmerksam machen möchte. Es saugt, schluckt und fühlt Berührungen. Die Bewegungsfähigkeit von Armen und Beinen wird erst deutlich später ausgereift sein. So wird es noch einige Monate dauern, bis das Kind sich aus der Bauch- in die Rückenlage drehen bzw. krabbeln oder laufen kann. Von Geburt an sind wir neurobiologisch also nicht nur auf Essen und Schlafen ausgerichtet, sondern auch auf Kommunikation und Kontakt.

In der zweiten Qualität reagiert unser Gehirn auf Angst, Gefahr und ungelöste Probleme. Dadurch wird die
Aktivität des ventralen Vagus und des SSE geschwächt und der Sympathikus aktiviert. Wenn der Modus des Sympathikus mit seinen Möglichkeiten des Kämpfens oder Flüchtens zu keiner Lösung führt bzw. die innere Erregung und Wahrnehmung von Bedrohung unerträglich $\mathrm{zu}$ werden beginnt, tritt die dritte Qualität ein.

Im dritten Fall fällt der Körper auf das entwicklungsgeschichtlich älteste System des ANS zurück, den dorsalen Vagus. Wie bei den Reptilien im Moment der Orientierung kommt es zur Erstarrung, zum Totstellreflex mit dem abrupten Abfall von Herzschlag und Blutdruck. Der Zustand ist verbunden mit maximaler Erregung und gleichzeitig einer Distanz zu den Gefühlen, einer Dissoziation.

Unter normalen Umständen ist der dorsale Vagus durch den Sympathikus und dieser wiederum durch den ventralen Vagus gebremst. Immer kontrolliert also das entwicklungsgeschichtlich jüngere System das ältere. Nur im Notfall springt das ältere System sozusagen in die Bresche (Prinzip von Jackson [11]).

Jedoch auch im Normalfall hat der dorsale Vagus beim Menschen Funktionen. Er steuert die Organe unterhalb des Zwerchfells, also Magen und Darm. Der «gesunde» dorsale Vagus lenkt also die Verdauungsorgane und wird auch im Schlaf aktiver.

\section{Polyvagaltheorie, Hören, Tomatis und Caruso}

Wir sind ausgegangen von der goldenen Stimme von Caruso und den Untersuchungen von Dr. Tomatis über die Zusammenhänge von Stimme, Psyche und Gehör und kommen nun dahin zurück. Das besondere Hören mittlerer und hoher Töne erzeugt einen Reichtum an Obertönen in der Stimme, und dieses höhenbetonte Hören wird wesentlich durch die Spannung der Mittelohrmuskeln mit bewirkt. Die Steuerung der Mittelohrmuskeln erfolgt weitgehend un- 
willkürlich durch das ANS. Wie ist diese Steuerung nun aus der Sicht der Polyvagaltheorie einzuordnen?

Mit den Säugetieren und dem Menschen wird das Hören in den Dienst der Kommunikation gestellt. Prinzipiell verläuft der Vorgang des Hörens folgendermassen: Schallwellen werden durch die Ohrmuscheln aufgefangen und treffen auf das Trommelfell. Das Trommelfell mit den drei Gehörknochen ist Teil des luftgefüllten Mittelohrs. Die drei Gehörknochen übertragen die Schalldruckwellen über eine kleine Membran zum Innenohr. Im Innenohr in Form einer Schnecke mit zweieinhalb Windungen sitzen in sehr salzhaltiger Flüssigkeit die Hörsinneszellen, die Haarzellen. Die Schalldruckwelle läuft durch das Innenohr und schert die Härchen der Haarzellen ab. Die Haarzelle reagiert auf diese Abscherung ihrer Härchen mit einer elektrischen Entladung, die über den Hörnerv zum Gehirn weitergegeben wird. Im Mittelohr sitzen die bereits erwähnten zwei Mittelohrmuskeln, der Trommelfellspanner und der Stapediusmuskel. Das Mittelohr ist im Prinzip ein Hebelwerk, das den Schalldruck etwa dreissigfach verstärkt. Reguliert wird es durch diese beiden Muskeln. Eine starke Spannung vor allem des Stapediusmuskels schützt das Innenohr und die Haarzellen vor zu starker Lautstärke vor allem im Bereich tiefer Töne (Stapediusreflex). Der Trommelfellspanner sensibilisiert durch Straffung des Trommelfells die Schallleitung.

In der klassischen Auffassung der Hals-Nasen-Ohren-Medizin erfolgt die Regulation dieser Muskeln weitgehend reflektorisch, wie wenn man mit der Hand auf die heisse Herdplatte fasst und diese reflexartig zurückzieht, noch bevor man überhaupt bewusst realisiert hat, dass diese heiss ist. Nach Tomatis werden die Muskeln jedoch auch wesentlich durch emotionale Prozesse mit beeinflusst $[1,12]$. Wenn unsere Gefühlszentren im Gehirn sagen «Ich möchte diese Stimme hören, weil sie so melodiös und warm klingt», werden unwillkürlich die Mittelohrmuskeln gestrafft. Die menschliche Stimme hat ihre Frequenzanteile mehr im mittleren und höheren Tonbereich. Durch Spannung der Mittelohrmuskeln und des Trommelfells werden eben diese mittleren und hohen Töne verstärkt zum Innenohr geleitet. Die Stimme hebt sich hervor aus den tieferen Umgebungsgeräuschen. (Dies erklärt, warum wir in einem wilden Stimmengewirr - bei normaler organischer Hörfähigkeit - die Stimme unserer Gesprächspartner gut hervorheben können. Unser Bauch und Gehirn sagen «ja» zur Stimme des Gegenübers und bringen unsere Mittelohrmuskeln zur guten Spannung.)

Stephen Porges ordnet die Funktion der Mittelohrmuskeln explizit in diesen Zusammenhang ein. Sie sind Teil des SSE. Zusammen mit dem ventralen Vagus ist ihre Spannung immer dann gegeben, wenn unser Gehirn der Meinung ist, dass wir uns in einem Zustand von Sicherheit und Wohlbehagen befinden. Auf die Kindesentwicklung bezogen heisst dies, dass die Spannung der Mittelohrmuskeln sehr wahrscheinlich mit einem Erleben von sicherer Bindung verknüpft wird.

In diesem Sinn berührt uns die Stimme von Caruso nicht nur wegen ihrer Fülle und ihres aussergewöhnlichen Timbres, sondern auch, weil unser ANS sie einem Zustand von wohliger Sicherheit und lebendiger Freude, der Qualität des ventralen Vagus, zuordnet.

In der Systemischen Hörtherapie, aufbauend auf den Grundlagen von Dr. Tomatis, führen wir spezielle Hörtests durch, eine besondere Form der Hörschwellenaudiometrie. In diesen Hörtests wird die Empfindlichkeit des Hörens für eine Vielzahl von Tönen bestimmt. Sehen wir in diesem Hörtest die beschriebene besondere Hervorhebung mittlerer und hoher Töne, so verstehen wir dies als ein Zeichen sowohl guter Führung der eigenen Stimme als auch von guter Kontakt- fähigkeit und sicherer Bindung. In der auf den Hörtest aufbauenden Hörtherapie hören die Klienten Musik von Mozart über Spezialkopfhörer. Die Musik wird dabei immer durch Klangwandler verändert. Ein Grundprinzip der Hörtherapie ist eine «musikalische Mikrogymnastik» des muskulären Mittelohrsystems durch die sogenannte «Klangwippe». Hierbei wird die Musik in einem dauernden Wechsel von Tiefenbetonung und dann wieder Höhenbetonung gehört. Die Tiefenbetonung wirkt auf die Mittelohrmuskeln entspannend. Mit einem dann etwas erschlafften Trommelfell werden tiefe Töne bevorzugt wahrgenommen. Die Patienten reagieren auf diese Veränderung in aller Regel mit einer Tiefenentspannung. Dann erfolgt immer wieder die Aufhellung der Musik, ein Reiz zur Straffung des Mittelohrmuskelsystems. Die hohen Töne werden bevorzugt weitergeleitet; die Patienten reagieren typischerweise mit einer Wachheit.

Im Sinne der Polyvagaltheorie kann angenommen werden, dass durch die Klangwippe eine pulsatorische Aktivierung vor allem auch des ventralen Vagus erzielt wird. Die Höhenbetonung der Klangwippe aktiviert in dieser Sicht den ventralen, sozialen Vagus. Die Tiefenbetonung stimuliert den «guten» dorsalen Vagus, der im Erleben von Sicherheit eine «liebevolle Ruhe» vermittelt. Diese Aussage ist sehr bedeutsam, denn viele weitere aktuelle Forschungen weisen darauf hin, dass eine Aktivierung des Vagus, im Besonderen des ventralen Vagusasts, eine Haltung von Achtsamkeit und Meditation fördern. Die «musikalische Mikrogymnastik» der Klangwippe ist also weit mehr als ein Muskeltraining. Sie wirkt auf das ANS und kann zu einem Erleben von Ruhe, Loslassen und Wohlbehagen führen. Dies kann sehr erfolgreich bei Patienten mit Burn-out, Ängsten, Depressionen oder Tinnitus angewandt werden, und zwar gerade auch auf die körperlichen Aspekte dieser Erkrankungen. Als Beispiel 
seien noch einmal die zu eng angezogenen «Zügel» des Herzens bei Depressionen genannt, die durch die Anwendung der Hörtherapie gelöst werden können.

Werden diese Zügel wieder locker gelassen und ist der jeweilige individuelle Rhythmus von Aktivität und Ruhe wieder gefunden, dann kann auch Spannkraft, Timbre und Freude in eine angespannte oder brüchige Stimme kommen. Indem das «Ich» sich wieder findet, kann die Begegnung im «Du» ebenso möglich werden.

\section{Literatur}

1 Tomatis AA: L’oreille et la vie. Itinaire d'une recherche sur l'audition, la langue et la communication. Paris, Robert Laffon, 1977. (Deutsch: Das Ohr und das Leben. Erforschung der seelischen Klangwelt. Düsseldorf, Walter, 1995.)

2 Dieroff H-G: Lärmschwerhörigkeit. Jena, Gustav Fischer, 1994, p 46.

3 Porges S: Die Polyvagaltheorie. Neurophysiologische Grundlagen der Therapie. Paderborn, Junfermann, 2010.

4 Sympathikus. http://de.wikipedia.org/wiki/ Sympathikus.

5 Cannon WB: The mechanism of emotional disturbance of bodily functions. N Engl J Med 1928;198:877-884.

6 Darwin C: The Expressions of the Emotions in Man and Animals. London, John Murray, 1872.
7 Schnack G: Der Grosse Ruhe-Nerv. Soforthilfen gegen Stress und Burn-out. Freiburg i.Br., Kreuz, 2012.

8 Bauer J: Das Gedächtnis des Körpers. Wie Beziehungen und Lebensstile unsere Gene steuern. München, Piper, 2008.

-9 Kreuzer PM, Vielsmeier V, Langguth B: Chronischer Tinnitus - eine interdisziplinäre Herausforderung. Dtsch Ärztebl Int 2013;110:278-284.

10 Levine P: Waking the Tiger. Healing Trauma. Berkeley, CA, North Atlantic Books, 1997. (Deutsch: Traumaheilung. Das Erwachen des Tigers. Unsere Fähigkeit, traumatische Erfahrungen zu transformieren. Essen, Synthesis, 1998.)

11 Jackson JH: Evolution and dissolution of the nervous system; in Taylor J (ed): Selected Writings of John Hughlings Jackson, vol 2. New York, Basic Books, 1958, pp 45-75.

12 Tomatis AA: La nuit utérine. Paris, Editions Stock, 1981. (Deutsch: Der Klang des Lebens. Hamburg, Rowohlt, 1995.) 